\title{
Reproduction of the evolution of the liquid front profile in inhomogeneous nanoporous media
}

\author{
D. S. Golovina, S. A. Chivilikhin \\ ITMO University, Kronverkskiy, 49, St. Petersburg, 197101, Russia \\ dheaddd@gmail.com, sergey.chivilikhin@gmail.com
}

PACS 68.03.-g

DOI 10.17586/2220-8054-2017-8-5-567-571

\begin{abstract}
In recent years possible applications of nanoporous materials in biophysics and biomedicine have become a topic of intense scientific interest. One of the main problems in this field is that of transport processes in inhomogeneous nanoporous media. Another one is the reproduction of the specific evolution of the liquid front profile, observed in one medium, in another medium. In this paper, we present a model which simulates two-dimensional liquid front propagation in inhomogeneous nanoporous media; we also propose a method to evaluate the parameters of the nanoporous medium required for reproduction of the given liquid front propagation.
\end{abstract}

Keywords: inhomogeneous nanoporous media, transport processes, liquid front propagation.

Received: 15 September 2017

Revised: 9 October 2017

\section{Introduction}

In last decade, nanoporous materials have become a point of considerable scientific interest. They are widely studied and used in biophysics and biomedicine; nanoporous materials are applied in drug delivery [1,2], tissue engineering [3], as biosensors [4], have great potential for usage in pharmacology [5], etc.

One significant problem in this field is the analysis of transport processes, which are considered to be more complex than in porous media, especially if nanoporous media in question are inhomogeneous [6]. Also, in biophysics and biomedicine, it is often necessary to reproduce in synthesized non-organic nanoporous medium the specific pattern of evolution of the liquid front profile observed in organic nanoporous medium.

The purpose of our work is to propose a model for simulation of the two-dimensional liquid front propagation in inhomogeneous nanoporous media and a method to evaluate the parameters of the medium in which a liquid will flow in a specific manner.

\section{Mathematical model and its numerical realization}

In this paper, we consider the flow in a thin nanoporous slab with thickness $<1 \mathrm{~mm}$. We introduce the Cartesian coordinates along the slab - horizontal coordinate $x$ and vertical coordinate $y$. The width of slab is $L$, the vertical coordinate of the boundary of the liquid front is $H(x, t)$ - see Fig. 1.

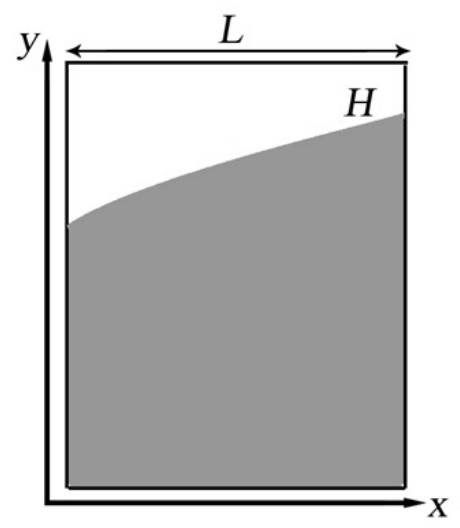

FIG. 1. Thin nanoporous slab. Gray area is the part of the medium permeated by liquid 
While liquid flow permeates through a medium, the capillary forces in the liquid surface create the pressure $P_{H}=-\frac{2 \sigma}{R}$, where $\sigma$ is the surface tension coefficient of the liquid and $R$ is the pore radius. The process of flowing also creates the pressure in the volume of the propagating liquid.

The proposed mathematical model for describing the two-dimensional propagation of the liquid in inhomogeneous nanoporous media is based on the approximation of Darcy's law $\vec{v}=-\gamma \nabla P$, where $\vec{v}$ is the velocity of the flow and $P$ is the pressure in the liquid, and on the continuity equation for the incompressible flow $\nabla \vec{v}=0$. In the two-dimensional case, the combination of these equations gives us the linear differential equation:

$$
\nabla(\gamma \nabla P)=0
$$

with boundary conditions - definite pressure on the bottom of the thin slab $(y=0)$ and on the boundary of the liquid front $(y=H(x, t))$ plus the impermeability of the sidewalls $(x=0, x=L)$ :

$$
\begin{aligned}
& \left.P\right|_{y=0}=0 \\
& \left.P\right|_{y=H}=-\frac{2 \sigma}{R} \\
& \left.\frac{\partial P}{\partial x}\right|_{x=0}=\left.\frac{\partial P}{\partial x}\right|_{x=L}=0 .
\end{aligned}
$$

The coefficient $\gamma$ in (1) describes the permeability of the medium [7]:

$$
\gamma=\frac{\Omega R^{2}}{8 \mu},
$$

where $\Omega$ is the porosity of medium (volume ratio of the pore space to the total volume of the material), $R$ is the average pore radius and $\mu$ is the dynamic viscosity coefficient of the fluid.

The coefficient $\gamma$ describes the structural properties of the nanoporous medium. By changing $\gamma$ values in every grid point, we take into consideration the changes in porosity or/and pore size in the medium and their influence on the shape and transition of the boundary of the front.

On the whole, the numerical calculation procedure consists of two parts. First, by solving linear differential equation (1) we obtain the pressure distribution in the part of the medium which has been permeated by liquid. While pressure and distance values for grid points which are remote from border can be obtained directly from adjacent grid points, calculation of those values for grid points which are adjoined to the border requires an interpolation procedure.

Once the pressure distribution is obtained, we shift the boundary of the front on the basis of the pressure derivatives. Since we consider inhomogeneous nanoporous media, capillary pressure $P u$ changes with the motion of the front and thus, must be recalculated after each iteration.

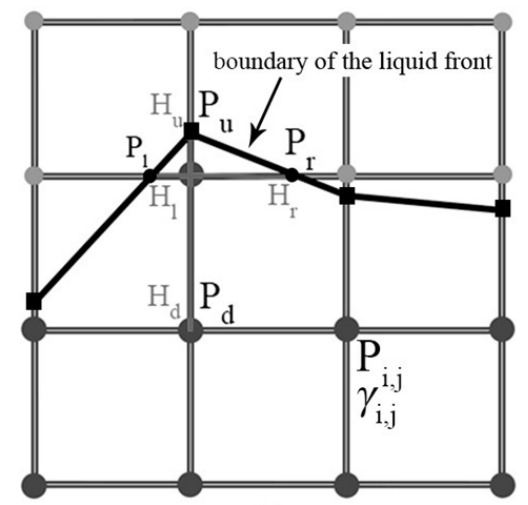

(a)

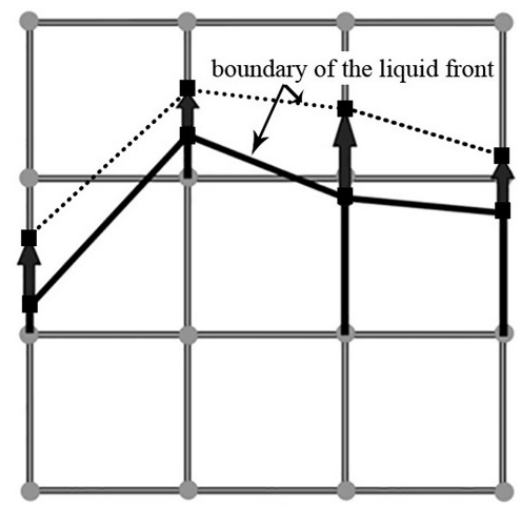

(b)

FIG. 2. (a) The numerical calculation procedure, step 1: solving linear differential equation (1) by finite-difference method. Pressure values $P_{r}, P_{l}$ and distance values $H_{u}, H_{d}, H_{r}, H_{l}$ are obtained by linear interpolation between central grid point and upper, lower, right and left grid points accordingly with $P_{u}$ being the capillary pressure and $P_{d}$ being the pressure obtained directly from lower grid point. (b) The numerical calculation procedure, step 2: translocation of boundary of the front 
Figure 2 shows the result of numerical simulation of the water $(\sigma=0.07 \mathrm{~N} / \mathrm{m}, \mu=0.8 \mathrm{mPa}$ s) propagation in nanoporous thin film $(20 \times 20 \mathrm{~mm})$ with constant porosity $\Omega=0.5$ and pore radius changing randomly from 10 to $13 \mathrm{~nm}$.

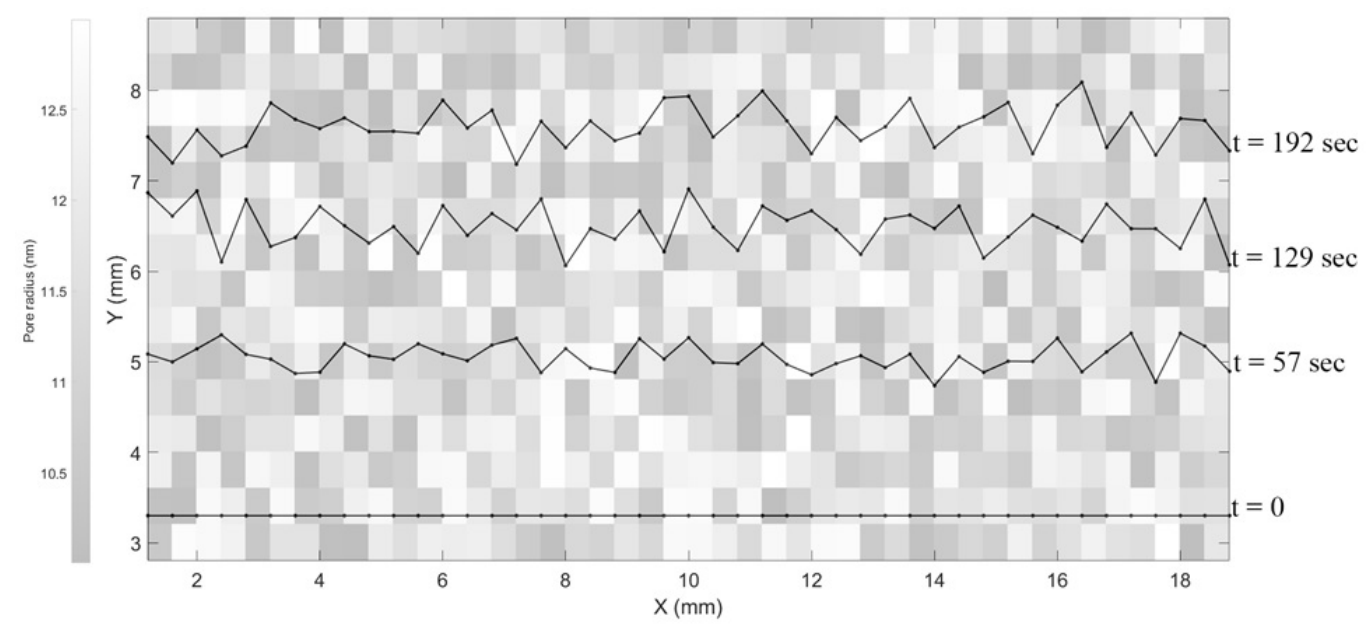

FIG. 3. Evolution of the water front profile in nanoporous thin film $(20 \times 20 \mathrm{~mm})$ with constant porosity $\Omega=0.5$ and pore radius changing randomly from 10 to $13 \mathrm{~nm}$. Darker areas on the background indicate the smaller pore radius and lighter ones, accordingly, larger

Figures 3, 4 show the simulations for the same scenario, only with porosity not being constant, but changing stepwise relative to $x$. $\Omega=0.2$ while $x<6.8 \mathrm{~mm}, \Omega=0.8$ while $6.8 \geq x<13.6 \mathrm{~mm}$ and $\Omega=0.2$ while $x \geq 13.6 \mathrm{~mm}$. As expected, the increase of porosity leads to additional rise of the front boundary, although while the porosity changes strictly stepwise, the deformation of the boundary of the front is gradually linear.

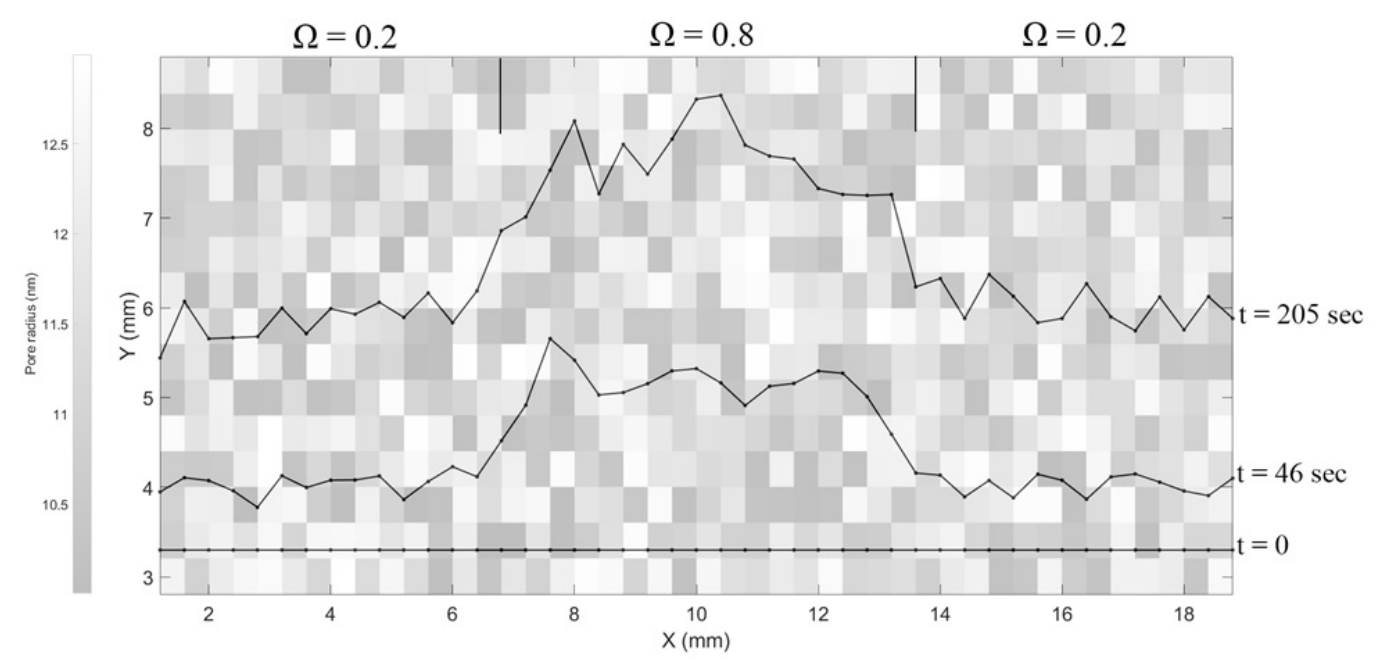

FIG. 4. Evolution of the water front profile in nanoporous thin film $(20 \times 20 \mathrm{~mm})$ with porosity changing stepwise from 0.2 to 0.8 and back to 0.2 and pore radius changing randomly from 10 to $13 \mathrm{~nm}$. Darker areas on the background indicate the smaller pore radius and lighter ones, accordingly, larger

\section{Evaluation of the parameters of the inhomogeneous nanoporous medium required for reproduction of the specific liquid front propagation}

On the basis of the model described above we propose a method of evaluating the parameters of the inhomogeneous nanoporous medium in which the liquid will flow as it is required. 
The porosity distribution or/and pore radius distribution in inhomogeneous nanoporous medium can be viewed as polynomial of some degree:

$$
R_{x, y}\left(\Omega_{x, y}\right)=A_{1,1} x^{n}+A_{1,2} y^{n}+A_{2,1} x^{n-1}+A_{2,2} y^{n-1}+\ldots+A_{n-1,1} x+A_{n-1,2} y+A_{n},
$$

where $A_{j, k}$ are the coefficients of the polynomial and $x, y$ are the coordinates of the medium.

Any set of the polynomials' coefficients correspond to a certain evolution of the liquid front profile. The task of finding suitable parameters reduces to the problem of function minimization; the coefficients of the polynomial are being adjusted until the difference between resulting evolution of the liquid front and sought-for one becomes minimal. As a measure of difference, we considered the standard deviation between the points of the reproduced boundary of the front and original one.

An example of such reproduction is shown in Fig. 5. The original evolution of the water front profile was modelled in nanoporous thin film $(40 \times 40 \mathrm{~mm})$ with constant porosity $\Omega=0.5$ and pore radius increasing quadratically from $7 \mathrm{~nm}$ to $23 \mathrm{~nm}$ relatively to $x(\mathrm{~mm}): R(\mathrm{~nm})=9.38 \cdot 10^{-3} x^{2}+2.5 \cdot 10^{-2} x+7$. The reproduction process has been simulated for the $4^{\text {th }}$ degree polynomial - the resulting reproduced pore size distribution is $4^{\text {th }}$-degree increase from $7 \mathrm{~nm}$ to $28 \mathrm{~nm}$.

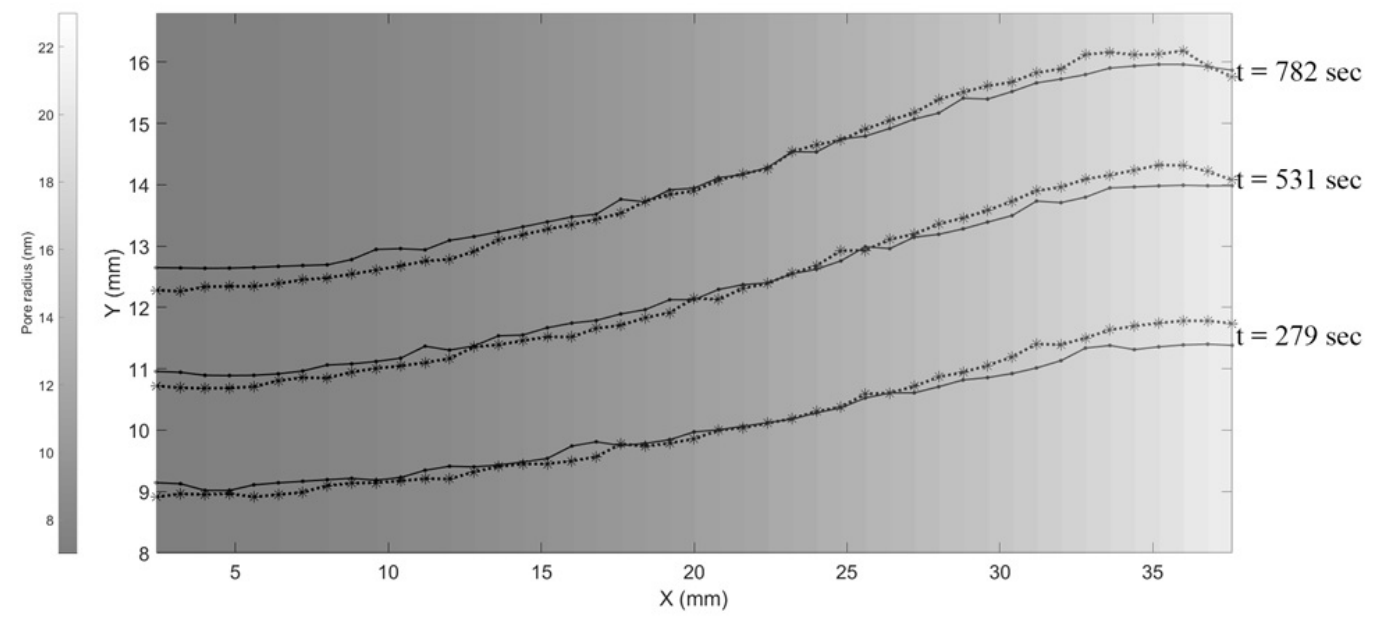

FIG. 5. The original evolution of the water front profile in nanoporous thin film with constant porosity $\Omega=0.5$ and pore radius increasing quadratically from $7 \mathrm{~nm}$ to $23 \mathrm{~nm}$ (point markers, solid line) and reproduced evolution front profile in thin film with the same porosity and pore radius distribution being $4^{\text {th }}$-degree increase from $7 \mathrm{~nm}$ to $28 \mathrm{~nm}$ (asterisk markers, dotted line). Darker areas on the background indicate the smaller pore radius and lighter ones, accordingly, larger

As can be seen, the results are in good correspondence with each other.

This method can also be used if the required porosity and pore radius distributions are known, but synthesis of such medium is impossible or if for analysis/modelling purposes, the calculation time is too large because of distribution complexity. If simpler distributions are required, this method can be used for finding an alternative solution, suited for the task at hand.

Figure 6 shows the original evolution of the water front profile in a thin nanoporous film $(40 \times 40 \mathrm{~nm})$ with constant porosity $\Omega=0.5$ and pore radius increasing cubically from $8 \mathrm{~nm}$ to $20 \mathrm{~nm}$ relative to $x$ (mm): $R(\mathrm{~nm})=13.67 \cdot 10^{-5} x^{3}+15.63 \cdot 10^{-4} x^{2}+2.5 \cdot 10^{-2} x+8$, which was considered original, and "reproduced" evolution of the water front profile in nanoporous thin film with porosity $\Omega=0.7$ and pore radius increasing linearly from $6 \mathrm{~nm}$ to $13 \mathrm{~nm}$ relative to $x$.

\section{Conclusion}

The mathematical model which describes the two-dimensional propagation of liquid in inhomogeneous nanoporous media has been presented along with its numerical realization. On the basis of this model a method of evaluating the parameters of the inhomogeneous nanoporous medium resulting in sought-for specific pattern of the liquid front propagation has been proposed. Several scenarios of reproduction of the specific evolutions of the liquid front profile have been presented - it has been shown that proposed method is effective for both 


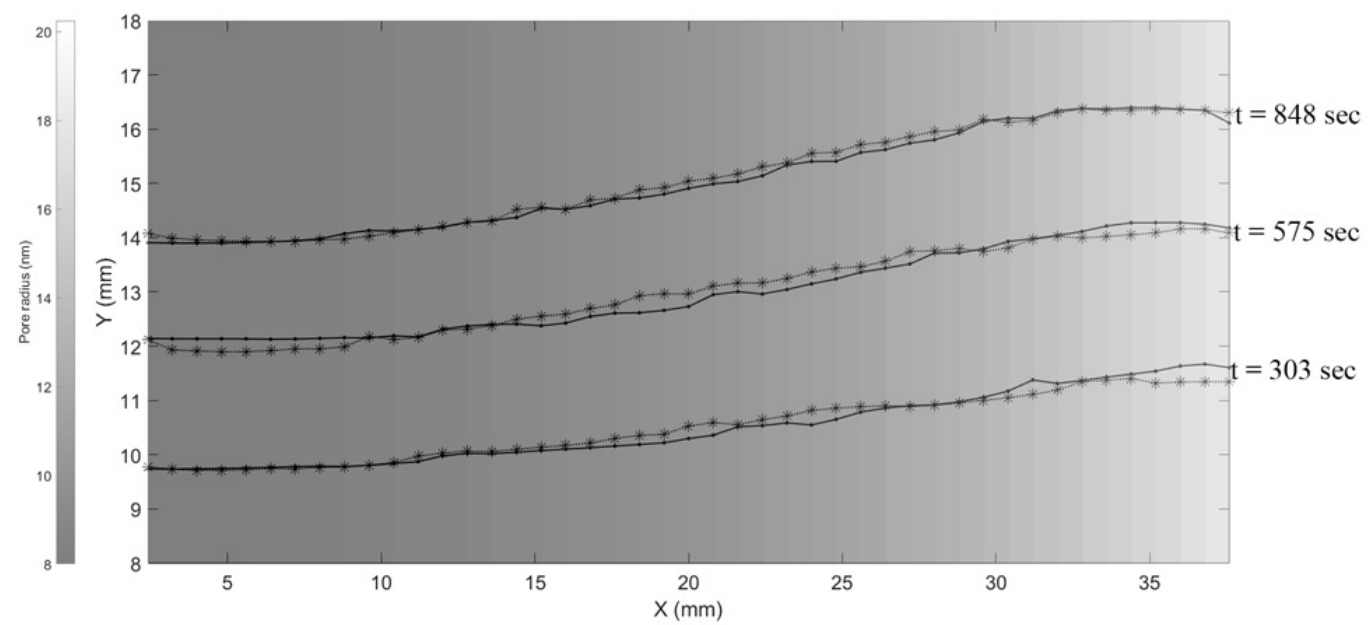

FIG. 6. The original evolution of the water front profile in nanoporous thin film with constant porosity $\Omega=0.5$ and pore radius increasing cubically from $8 \mathrm{~nm}$ to $20 \mathrm{~nm}$ (point markers, solid line) and reproduced evolution front profile in thin film with porosity $\Omega=0.7$ and pore radius increasing linearly from $6 \mathrm{~nm}$ to $13 \mathrm{~nm}$ (asterisk markers, dotted line). Darker background areas indicate smaller pore radius and lighter ones, accordingly, larger

evaluating the needed parameters as accurately as possible (which can be used for further mathematical analysis and modelling) and finding distributions which can be synthesized with greater ease at lower costs.

\section{References}

[1] Solomatin A.S., Yakovlev R.Yu., Efremenkova O.V., Sumarukova I.G., Kulakova I.I., Lisichkin G.V. Antibacterial activity of Amikacinimmobilized detonation nanodiamond. Nanosystems: physics, chemistry, mathematics, 2017, 8(4), P. 531-534.

[2] Sun C.Y., Qin C., Wang C.G., Su Z.M., Wang S., Wang X.L., Yang G.S., Shao K.Z., Lan Y.Q., Wang E.B. Chiral Nanoporous MetalOrganic Frameworks with High Porosity as Materials for Drug Delivery. Advanced Materials, 2011, 53, P. 5629-5632.

[3] Cardea S., Pisanti P., Reverchon E. Generation of chitosan nanoporous structures for tissue engineering applications using a supercritical fluid assisted process. The Journal of Supercritical Fluids, 2010, 54, P. 290-295.

[4] Hu K, Lan D, Li X and Zhang S. Electrochemical DNA Biosensor Based on Nanoporous Gold Electrode and Multifunctional Encoded DNA-Au Bio Bar Codes. Analytical Chemistry, 2008, 80(23), P. 9124-9130.

[5] Almjasheva O.V., Garabadzhiu A.V., Kozina Yu.V., Litvinchuk L.F., Dobritsa V.P. Biological effect of zirconium dioxidebased nanoparticles. Nanosystems: physics, chemistry, mathematics, 2017, 8(3), P. 391-396.

[6] Conner W.C., Fraissard J. Fluid Transport in Nanoporous Materials: Proceedings of the NATO Advanced Study Institute, held in La Colle sur Loup, France, 16-28 June 2003 (Nato Science Series II:). Springer, 2006, 686 p.

[7] Scheidegger A.E. The Physics of Flow Through Porous Media. University of Toronto Press, Toronto, 1974,353 p. 